\title{
Editorial. \\ La comunicación y la crisis sanitaria: un tiempo de cambios acelerados
}

\author{
Javier Marzal-Felici \\ Universitat Jaume I \\ Andreu Casero-Ripollés \\ Universitat Jaume I
}

\section{Referencia de este artículo}

Marzal-Felici, Javier y Casero-Ripollés, Andreu (2020). Editorial $n^{\circ} 20$. La comunicación y la crisis sanitaria: un tiempo de cambios acelerados. En: adComunica. Revista Científica de Estrategias, Tendencias e Innovación en Comunicación, $\mathrm{n}^{\mathrm{o}}$ 20. Castellón: Universitat Jaume I, 11-18. DOI: http://dx.doi.org/10.6035/21740992.2020 .20 .1 .

\section{La comunicación y la crisis sanitaria: un tiempo de cambios acelerados}

Sin lugar a dudas, la crisis sanitaria provocada por la pandemia del virus SARS$\mathrm{CoV}-2$ ha irrumpido de manera traumática en nuestras vidas, con efectos terribles en la salud física y mental de todos nosotros, en la economía, en la educación, en la forma de relacionarnos con la gente, etc. Ya son más de 15 millones de infectados y más de 620.000 muertos, a fecha de 24 de julio de 2020, y las cifras no dejan de crecer. En España, los diagnosticados por COVID-19 ascienden a más de 270.000 
personas, y el número de personas fallecidas es superior a 28.400. Desde el fin de la declaración del estado de alarma, el número de brotes y de personas infectadas no ha dejado de aumentar, y se teme muy seriamente la aparición de una segunda oleada que puede llegar incluso antes del otoño. En muy poco tiempo, nos hemos visto obligados a teletrabajar, a impartir clases de forma online, a conciliar la atención a niños y mayores con la nueva realidad laboral, a mantener el contacto con familiares y amigos a través de las redes sociales y de las herramientas telemáticas, a aislarnos socialmente para detener la escalada de contagios.

Y no podemos olvidar a los millones de personas que en estos meses han perdido sus trabajos, a las miles de empresas que han tenido que suspender su actividad o, incluso, que han tenido que cerrar. También debemos recordar el trabajo extraordinario que ha realizado el personal sanitario y los trabajadores de otros servicios esenciales, desde las fuerzas de seguridad hasta los trabajadores de sectores tan vitales como la alimentación, el transporte, la energía, etc. En efecto, nuestras vidas han sufrido una profunda transformación que nadie podía sospechar el pasado mes de enero, cuando publicábamos el número 19 de la revista adComunica, con una temática central dedicada a las formas del «efecto documental» en el cine contemporáneo. Parece que estemos viviendo una ficción cinematográfica que describía, con una precisión descarnada, Contagio (Contagion, Steven Soderbergh, 2011), confirmando la conocida expresión de que «la realidad supera la ficción».

Pero los cambios también han irrumpido bruscamente, y de forma profunda, en el campo de la comunicación. No en vano, la declaración del estado de alarma convertía «oficialmente» a los medios de comunicación en «servicios esenciales». Hemos podido constatar, como ha señalado Andreu Casero-Ripollés, que la pandemia provocada por la COVID-19 ha favorecido la reconexión de las audiencias con los medios de comunicación tradicionales, especialmente, la televisión, de tal modo que las noticias se han convertido en un recurso muy valioso para la ciudadanía desde el inicio de esta crisis sanitaria [...] por lo que analizar las dinámicas de consumo informativo resulta altamente relevador tanto por su capacidad de transformar el sistema mediático como por su incidencia democrática» (CaseroRipollés, 2020). En este reciente estudio, basado en un análisis exploratorio a partir de datos secundarios de las encuestas online del Pew Research Center's American Trends Panel norteamericano, mediante la comparación de cifras anteriores y posteriores a la pandemia, se constata que en situaciones críticas de alta complejidad y riesgo para la vida humana, como ocurre con la crisis por el coronavirus, las audiencias sitúan la búsqueda de información y el seguimiento de noticias como actividad clave, con un incremento de un 34\% en el consumo de noticias y con un 92\% de adultos de los Estados Unidos (EEUU) encuestados, que accedieron con frecuencia a noticias sobre la pandemia. El análisis del consumo de noticias ha permitido constatar el auge imparable de las redes y, lo más llamativo, la enorme relevancia de la televisión nacional en abierto y de la televisión por cable, así 
como un aumento muy notable de la confianza en estos medios, en contraste con las redes sociales, que generan mayor desconfianza por la circulación más alta de fake news y mensajes de odio.

En estos meses de aislamiento social forzoso, cabe subrayar el notable incremento de producción científica en el campo de las ciencias de la comunicación, en especial en lo referente al estudio de los efectos de la crisis sanitaria en la comunicación, desde las más variadas perspectivas. Entre los numerosos trabajos publicados, se pueden destacar: el estudio de Jordi Xifra sobre los efectos de la pandemia del COVID-19 en la práctica de la comunicación corporativa y, en especial, en la comunicación interna y la responsabilidad social corporativa (Xifra, 2020); el análisis de la presencia de la imagen real del SARS-CoV-2 en los medios de comunicación, cuya falsedad constituye una manifestación más de la infodemia que estamos sufriendo (Andreu-Sánchez, Martín-Pascual, 2020); o los numerosos trabajos sobre la relevancia de la comunicación en la gestión de la crisis del COVID-19, y sus efectos políticos, desde múltiples perspectivas (López-García, 2020; Costa-Sánchez, López-García, 2020; entre otros).

Durante esta etapa de pandemia, también hemos sido testigos de la importancia que tiene el entretenimiento y la ficción audiovisual, para acompañar a las personas en estos tiempos de confinamiento total y parcial, según sus etapas de desarrollo. En efecto, los medios de comunicación han sido una compañía muy importante para millones de personas en todo el mundo, y han contribuido a reforzar el sentimiento de pertenencia a una comunidad, permitiendo constatar cómo la comunicación es una actividad que tiene una profunda raíz antropológica, que nos define como especie humana (Duch y Chillón, 2012). Pero la crisis del coronavirus también ha multiplicado la capacidad de las redes sociales para expandir la desinformación, las falsedades y los bulos, poniendo de manifiesto cómo, junto a la pandemia del coronavirus, se ha extendido otra pandemia, también altamente letal: la epidemia de la desinformación, que ha inundado las redes sociales de todos tipo y condición, y que exige la aplicación de medidas preventivas, de una nueva dieta mediática (Sampedro, 2018), en especial en los campos social y educativo.

\section{La comunicación que viene: vías y tendencias de futuro}

No puede ser más oportuno el tema monográfico que se había programado hace más de año y medio para este número 20: «la comunicación que viene: vías y tendencias de futuro», que han coordinado los profesores Xosé López-García (Universidad de Santiago de Compostela), Carlos Toural (Universidad de Santiago de Compostela) y Luis Cárcamo Ulloa (Universidad Austral de Chile).

En efecto, la crisis sanitaria del COVID-19 ha acelerado vertiginosamente el avance hacia la sociedad digital, como consecuencia de la obligada adaptación al teletrabajo, en todas sus modalidades. Y cabe destacar que, en general, se ha producido 
un salto cualitativo, y hemos mejorado sustancialmente nuestras competencias digitales en unos pocos meses, si bien nos queda mucho camino por recorrer. Al mismo tiempo, hemos visto cómo la gestión de grandes bases de datos se ha convertido en esencial para avanzar hacia al control de la pandemia y, también, para el desarrollo del periodismo de datos que ahora mismo es fundamental para analizar y comprender cómo circula la información, cómo se genera y cómo se manipula en muchos casos. El contexto de la pandemia también está acelerando el desarrollo de la inteligencia artificial aplicada a los procesos comunicativos, no sólo en el contexto informativo, sino también en el terreno del entretenimiento audiovisual, para conocer los hábitos de consumo de la audiencia, así como en el campo publicitario, en pleno auge del marketing digital.

Todos los ciudadanos y ciudadanas nos hemos convertido en generadores de valiosa información que sólo las grandes corporaciones multinacionales de telecomunicaciones, los llamados GAFAM -Google, Apple, Facebook, Amazon, Microsoft - son capaces de procesar y de utilizar para sus intereses económicos y comerciales. La llamada «transición digital» está avanzando, pues, a una velocidad vertiginosa, coincidiendo además con el «segundo dividendo digital», una reordenación del espacio radioeléctrico que busca dejar sitio para la implantación de las nuevas redes de telecomunicación 5G, que multiplica por 10 la velocidad de las actuales redes de 4G, lo que permitirá aumentar exponencialmente el número de dispositivos conectados. Esta revolución tecnológica, unida también al aumento exponencial de la capacidad para procesar información con las últimas generaciones de procesadores informáticos, abre un horizonte de posibilidades muy amplio en el campo del acceso de la ciudadanía a la información y al entretenimiento, lo que plantea grandes desafíos para nuestra sociedad, cada vez más global. Los medios de comunicación - públicos y privados - han tenido que avanzar a marchas forzadas hacia la digitalización y refuerzo de sus plataformas multimedia. En este contexto, los medios de comunicación públicos, en especial, están obligados a ofrecer sus contenidos de forma síncrona pero también online, por lo que se está acelerando la convergencia entre prensa, radio y televisión, todo alojado en los sitios web de las corporaciones. Sin duda, la pandemia por el coronavirus está suponiendo un desafío sin precedentes para los profesionales de la comunicación.

\section{Medios de comunicación públicos y universidades: un maridaje necesario}

En el editorial del último número de la revista adComunica, de enero de 2020, nos hacíamos eco de una declaración conjunta de diferentes organizaciones y colectivos sociales, académicos y profesionales, denominada «Consenso y Concurso Público. Una solución democrática a la parálisis de RTVE», que apoyaban organizaciones como Teledetodos, la Asociación de Usuarios de la Comunicación (AUC), la Asociación de Emisoras Municipales y Ciudadanas de Andalucía de Radio y Televisión 
(EMARTV), la Red de Medios Comunitarios (ReMC), la Unión Latina de Economía Política de la Información, la Comunicación y la Cultura (ULEPICC), la Associació Ciutadania i Comunicació (ACICOM), la Federación de Sindicatos de Periodistas (FESP), entre otras. Explicábamos que los medios públicos están llamados a jugar un papel fundamental para compensar los fuertes desequilibrios que se dan en el sistema mediático actual, porque las profundas transformaciones - tecnológicas, económicas, políticas, culturales, etc.—, multiplicadas por la crisis del COVID-19, sitúan a los medios de comunicación públicos en un agente fundamental para la lucha contra la desigualdad social y cultural, en un contexto político, mediático y cultural dominado por las fake news y la desinformación.

En nuestra constante reivindicación de la relevancia de los medios públicos para el sistema mediático, queremos comentar brevemente la que para nosotros es una noticia de enorme trascendencia: À Punt Mèdia ha decidido fijar su sede en Castellón en el parque científico de la Universitat Jaume I, como ha sido anunciado el lunes 27 de julio de 2020. La ubicación física de la corporación pública en la UJI no es un asunto menor, y es un caso único a nivel español. En un principio, podría parecer que los medios de comunicación públicos y la universidad pública son ámbitos profesionales diferentes. En efecto, en los 24 años de historia de la antigua RTVV apenas existió relación con las universidades públicas, sólo circunscrita a las prácticas de los estudiantes de los grados de comunicación. En julio de 2017, antes del inicio de las emisiones de À Punt, las cinco universidades valencianas firmaron un ambicioso convenio marco que fijaba una hoja de ruta de permanente colaboración entre los nuevos medios de comunicación públicos valencianos y las universidades, en los campos de la investigación, de la docencia y de la transferencia.

Desde 2013, la Unión Europea de Radiodifusión, que agrupa a cerca de 70 radiotelevisiones públicas de todo el mundo, declaró que los principios que inspiran la actividad de los medios de comunicación públicos son la universalidad, la independencia, la excelencia, la diversidad, la rendición de cuentas (gobernanza) y la innovación (UER, 2013, 2014a, 2014b, 2015). Podemos afirmar, sin ambages, que estos seis principios también inspiran la actividad científica y académica de las universidades públicas. En la última década, todavía sacudidos por el impacto de la crisis financiera de 2008, ahora agravada por la crisis sanitaria del coronavirus, los medios de comunicación públicos han ido constatando que la innovación es esencial para hacer posible una reconexión con la ciudadanía, en especial, con el público más joven. La innovación es un valor estratégico imprescindible en un entorno altamente competitivo, donde las grandes plataformas como Netflix, Amazon, Disney o Apple, entre otras, están ocupando el espacio comunicativo.

Los viejos principios que inspiraron el nacimiento de la BBC, hace 82 años, «informar, entretener y formar» siguen teniendo plena vigencia hoy en día. Pero para hacerlo posible en la era del consumo online y de la hegemonía de las redes 
sociales, es imprescindible apostar por la innovación. En los países del norte de Europa, la colaboración entre los medios de comunicación y las universidades en los más diversos campos es habitual desde hace décadas. Desde todos los ámbitos del conocimiento de la Universitat Jaume I - las ciencias teóricas y experimentales, las ingenierías, las ciencias de la salud, la economía, las ciencias jurídicas, las humanidades y las ciencias sociales, la educación, la comunicación, las filologías, etc.- , se pueden ofrecer contenidos y posibilidades de colaboración realmente enormes, porque en las universidades nos esforzamos por cultivar el talento y por estimular la creatividad y la innovación.

La ubicación de la sede de À Punt en Castellón se produce en el parque científico de la UJI, donde se han instalado empresas que tienen en su ADN la innovación y la atracción del talento empresarial, por lo que creemos que la elección es muy adecuada y permitente, en un momento como el actual, en el que estamos viviendo una profunda revolución digital.

Sin lugar a dudas, la reputación positiva de la Universitat Jaume I redundará en el cambio necesario de la reputación de À Punt, lastrada por la imagen negativa de RTVV, que ha enmascarado también las cosas positivas que aportaron los medios públicos a la sociedad valenciana. No podemos olvidar la enorme importancia que tiene À Punt como motor del sector audiovisual valenciano y de las industrias culturales del territorio (Soler y Marzal, 2015). Pero para que esta colaboración funcione será imprescindible que los empleados de À Punt, y también de las universidades públicas, mostremos un mayor engagement (Marín-Pérez, 2019) y trabajemos codo a codo para servir a la sociedad valenciana. No se nos escapa que nos aguarda un reto realmente formidable, por ser inédito e innovador, que exigirá más trabajo y dedicación. Es imprescindible aprender de los errores del pasado y, por ello, es necesario no olvidar otro agente fundamental en esta ecuación: el propio tejido productivo comunicativo y audiovisual valenciano, que aglutina numerosas empresas de los ámbitos de la información, de la comunicación corporativa, de la publicidad y del audiovisual, con quienes debemos trabajar estrechamente.

Por todo ello, no podemos más que felicitar a la rectora de la Universitat Jaume I, Eva Alcón Soler; a la dirección del parque científico de la UJI, Espaitec, formado por Juan Antonio Bertolín, el vicerrector de investigación y transferencia, Jesús Lancis, y el vicerrector adjunto de transferencia, innovación, David Cabedo; y al director general de À Punt Mèdia, Alfred Costa, y a su equipo directivo, por haber realizado esta apuesta por la innovación y la excelencia. Sin duda, un maridaje de instituciones públicas que posee un enorme valor estratégico para la economía, la sociedad y la cultura valencianas. 


\section{La Escuela de Revisores de las revistas de comunicación}

El pasado 5 de mayo de 2020 se presentó un sitio web dedicado a reivindicar el papel y profundizar en el conocimiento de una actividad esencial para las revistas científicas como es la figura del revisor de los artículos científicos, según el procedimiento del peer review. La iniciativa ha surgido de 15 de revistas científicas del campo de las ciencias de la comunicación, formado por Comunicar, El profesional de la Información, Comunicación y Sociedad, Cuadernos.info, Revista Latina de Comunicación Social, Communication \& Society, Palabra Clave, Revista de Comunicación, Fotocinema, Doxa Comunicación, Estudios sobre el Mensaje Periodístico, Revista Mediterránea de Comunicación, Index Comunicación, Communication Papers y, también, adComunica. El objetivo es ofrecer una reflexión semanal sobre la relevancia de las revisiones científicas, una actividad imprescindible para la sostenibilidad del sistema de las revistas en el campo de las Ciencias de la Comunicación. Sin la colaboración desinteresada de los revisores sería imposible la existencia de las revistas científicas. Cada semana un miembro del equipo editorial de las diferentes revistas señaladas publica en este blog una reflexión sobre la actividad de la revisión científica, con el objetivo de avanzar hacia la realización de revisiones de mayor calidad.

Asimismo, debemos hacer referencia a la reciente renovación del sello de calidad a adComunica por la Fundación Española de Ciencia y Tecnología (FECYT), en reconocimiento de la calidad de la edición científica, lo que es un orgullo para el Departamento de Ciencias de la Comunicación, impulsor de la revista. Finalmente, queremos agradecer el compromiso y generosidad de investigadores y profesionales, y del equipo de dirección de la revista adComunica que han participado en este número, y que contribuyen a fortalecer este proyecto editorial.

\section{Referencias}

Andreu-Sánchez, Celia; Martín-Pascual, Miguel-Ángel (2020). Fake images of the SARS-CoV-2 coronavirus in the communication of information at the beginning of the first COVID-19 pandemic. En: El profesional de la información, vol.29, ${ }^{\circ} 3$. Barcelona: EPI. doi: https://doi.org/10.3145/epi.2020.may.09

Casero-Ripollés, Andreu (2020). Impact of COVID-19 on the media system. Communicative and democratic consequences of news consumption during the outbreak. En: El profesional de la información, vol.29, n ${ }^{0} 2$. doi: https://doi. org/10.3145/epi.2020.mar.23

Costa-Sánchez, Carmen; López-García, Xosé (2020). Comunicación y crisis del coronavirus en España. Primeras lecciones. En: El profesional de la información, vol.29, $\mathrm{n}^{0} 3$. Barcelona: EPI. doi: https://doi.org/10.3145/epi.2020.may.04 
Duch, Lluís y Chillón, Albert (2012). Un ser de mediaciones. Antropología de la comunicación. Vol. 1. Barcelona: Herder.

López-García, Guillermo (2020). Vigilar y castigar: el papel de militares, policías y guardias civiles en la comunicación de la crisis del COVID-19 en España. En: El profesional de la información, vol.29, $\mathrm{n}^{\circ} 3$. Barcelona: EPI. doi: https://doi. org/10.3145/epi.2020.may.11

Marín-Pérez, Benjamín (2019). Convertir a los colaboradores en embajadores de la marca: plan de comunicación interna para la televisión autonómica valenciana À punt”. En: El profesional de la información, vol.28, $\mathrm{n}^{\circ} 5$. Barcelona: EPI. doi: https://doi.org/10.3145/epi.2019.sep.15

Sampedro, Víctor (2018). Dietética digital. Para adelgazar al Gran Hermano. Barcelona: Icaria Editorial.

Soler, María y Marzal, Javier (2015). La relevancia estratégica de RTVV en el sistema comunicativo valenciano y para el desarrollo económico, social y cultural de la Comunidad Valenciana. En: Marzal, Javier; Izquierdo, Jéssica y Casero, Andreu (eds.). La crisis de la televisión pública. El caso de RTVV y los retos de una nueva gobernanza. Barcelona, Bellaterra, Castellón, Valencia: Universitat Pompeu Fabra, Universitat Autònoma de Barcelona, Universitat Jaume I, Universitat, Universitat de València. Col·lecció Aldea Global.

Unión Europea de Radiodifusión (2013). Funding Public Service Media, executive summary-brochure. Ginebra: UER.

Unión Europea de Radiodifusión (2014a). Vision 2020. Connecting to a Networked Society. Ginebra: UER. Consultado el 28 de julio de 2020 en http://www3.ebu. ch/files/live/sites/ebu/ files/Knowledge/Publication\%20Library/EBU-Vision2020Full_report_EN.pdf.

Unión Europea de Radiodifusión (2014b). Empowering Society. Declaration on the Core Values of Public Service Media. Ginebra: EBU.

Unión Europea de Radiodifusión (2015). Public Service Media. Contribution to Society. Informe producido por el Media Intelligence Service de la EBU. Suiza: MIS-EBU.

Xifra, Jordi (2020). Comunicación corporativa, relaciones públicas y gestión del riesgo reputacional en tiempos del COVID-19. En: El profesional de la información, vol.29, nº2. Barcelona: EPI. doi: https://doi.org/10.3145/epi.2020.mar.20 